\title{
Fast marching and acoustic descriptors based method for fish proportion interpolation
}

\author{
Imen Karoui, Ronan Fablet and Jean-Marc Boucher \\ Telecom Bretagne, UMR CNRS lab-STICC 3192, UEB, France
}

\begin{abstract}
We propose a new method for the estimation of fish abundance from both acoustic data and some trawl hauls catches. In this work, we operate at a global level and we aim at estimating fish abundance from these images and not to identify the species of each school. We associate each trawl catch to the nearest acoustic image and we describe each image by a set of global statistical distributions estimated on it and related to each school parameters. Then, we use the fast marching algorithm, a front propagation based scheme to define a region of interest around each trawl associated image. The fast marching algorithm propagates each front initialized on the image associated to the trawl samples with a velocity proportional to the distance between the trawl image acoustic features and those of the image for which we want to estimate the fish abundance. Finally, the fish abundance of each image is estimated as a weighted sum of the abundances associated to each trawl. The weights are estimated from the propagation time given by the fast marching algorithm. The method is experimented on real and synthetic data.
\end{abstract}

\section{INTRODUCTION}

Fish abundance estimation is the cornerstone of ecosystem based fisheries. Trawl surveys are the most important fisheries data source used in stock assessment. The acoustic survey data, with its more resolved sampling structure, is used to establish a relationship between the acoustic and trawl data and to interpolate the fish abundance in zones where trawls are not available. Here, we aim at developing an automatic method for the estimation of the fish abundance. In the literature, we distinguish two main approaches:

1- School based approaches that extract school fish from the echograms and state the problem as the classification of these extracted fish schools $[1,2]$.

2- Global approaches that are stated at the echogram level and estimate the global fish abundance on a whole acoustic image (an echogram part) [3,4]. The most popular method is the algorithm AICASA (Acoustic Image Classification And Species Allocation) [3] that directly infers species mixing proportions at the echogram level from global features computed as global statistics on the echogram. The method is based on hierarchical classification of echograms, where each class refers to a trawled site or a group of trawled sites. The fish abundance of a given image is estimated as the proportion of the class to which it belongs.

Global methods are more appropriate than school based ones because trawled sites involve a fish mixture and the use of the echogram provides a direct relation between acoustic data and species proportions.

Our proposed method is a generalization of the AICASA method. As AICASA, we use global feature estimated on fish schools extracted from echograms (typically, 3 nautical milelong echograms) using MOVIES software [5]. But, unlike AICASA, we do not classify the echograms and attribute the same mixing proportions for all images belonging to the same class; we estimate the inference of species mixing proportions for each echogram as a weighted sum of the mixing proportions evaluated at trawled sites. The weights are computed according to a fast marching algorithm [6] based on a distance between echogram statistics. Second, in AICASA, no spatial regularization is enforced, whereas some spatial regularity is expected, this is a built-in feature of the proposed approach.

The proposed method is applied on synthetic and real data and evaluated in terms of mixing proportion estimation accuracy.

The presented paper is organized as follows: in Section II, we introduce the problem and in Section III we describe the proposed method. In section IV, we give some results on synthetic and real data and in Section $\mathrm{V}$ we draw some conclusions.

\section{PROBLEM STATEMENT}

Backscattered-acoustic signal is digitized providing acoustic samples that form the echogram. The continuous recorded echogram is divided into bins. The binning results in a set of acoustic images that covers the survey area (typically, 3 nautical mile-long echograms). Figure 1 shows an example of echograms.

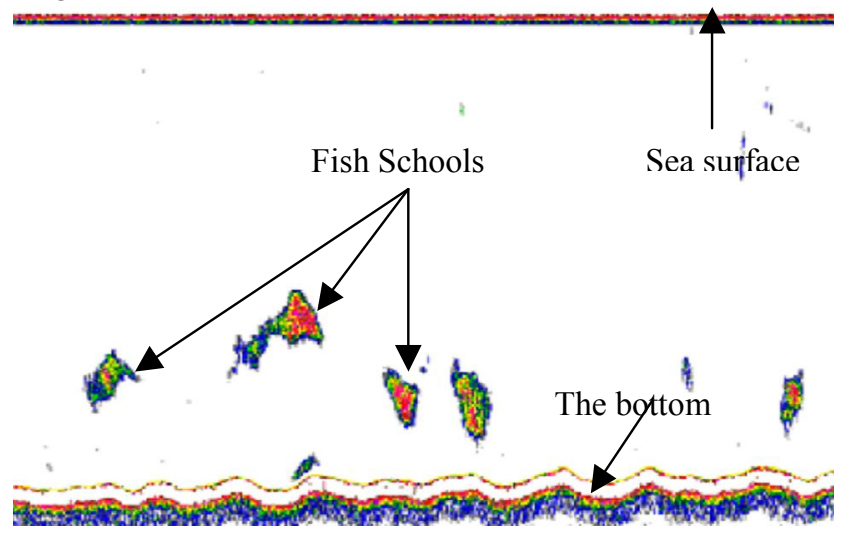

Figure 1. An echogram example (MOVIES software) 
Let $\Omega=\left\{I_{s}\right\}$ be this area to which we associate a spatial grid (see figure 2). Each grid point $I_{s}$ corresponds to an acoustic image (points in black in figure 2). In some grid points (presented by circles in figure 2), denoted by $\left\{I_{k}\right\}_{k=1: K}$ where $K$ is the total number of trawl catches, we have information about fish abundance through catch hauls. Each catch indexed by $k$ is mainly composed of a mixture of several species denoted by a vector $\Pi_{k}$ of $J$ elements, where $J$ is the number of fish species and each vector element $\Pi_{k}(j)$ represents the proportion of the species $j$ in the trawl catch $k$ such that:

$$
\sum_{j=1}^{J} \Pi_{k}(j)=1, \forall k
$$

$\Omega$

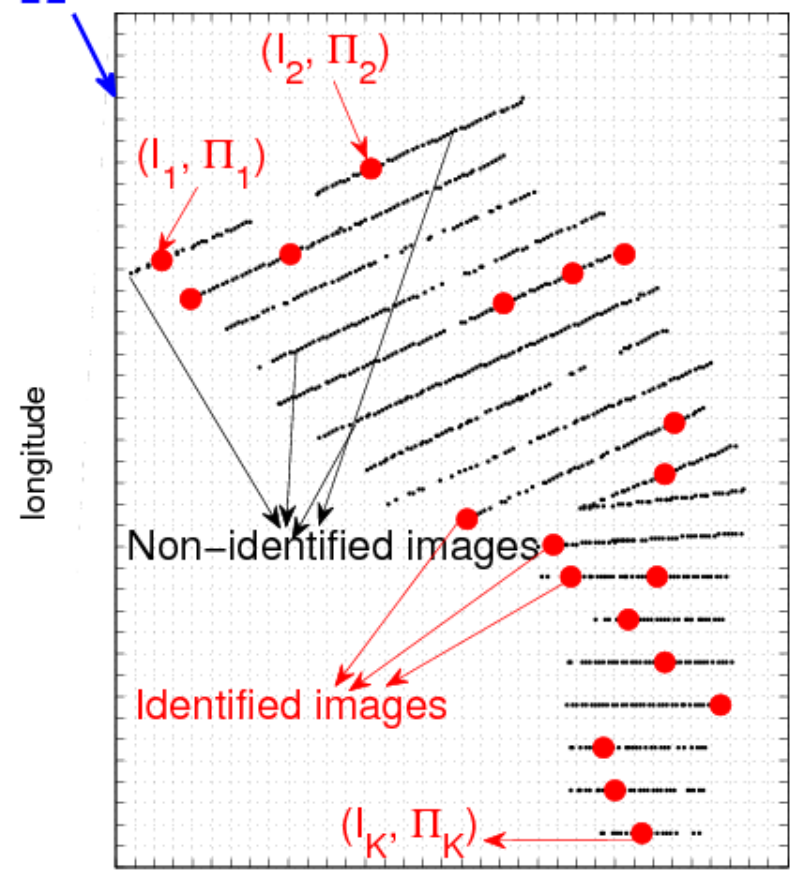

latitude

Figure 2. Notations

Each trawl catch is associated to a sonar observation for specific trawling areas (identified images). This association provides a mean for characterizing the information captured by the echosounder and the fish species presented in this area. Our aim is to exploit such training data to estimate the fish abundance $\left\{\Pi_{s}\right\}$ on non identified images $\left\{I_{s}\right\}$ based on their acoustic features. We estimate the non-identified proportions as a weighted sum of the identified ones:

$$
\Pi_{s}=\sum_{k=1}^{K} w_{k}\left(I_{s}\right) \Pi_{k}
$$

\section{METHOD DESCRIPTION}

The weights $\left\{w_{k}\right\}_{k=1: K}$ estimation is based on the hypothesis that acoustic image features are associated to the fish species mixture in the image and on the fact that fish abundance has some consistency in space. We propose an interpolation method based on the model given by equation (2) that computes the weight based on the spatial proximity of non-identified images $\left\{I_{s}\right\}$ to the identified ones $\left\{I_{k}\right\}_{k=1: K}$ and based also on the similarity of their acoustic content.

Acoustic content of each image is given by a set of acoustic parameters computed from fish schools extracted from each image. School objects were identified and extracted using MOVIES software [6]. From the extracted fish school we compute a set of feature distributions. As in [7], we use parameters belonging to four categories: school positions, school morphology, school density and the occupation of space by schools. In this application we used a set of 20 parameters proposed in [7]. Acoustic features are computed as the distribution of the different school features estimated on each image (mainly 100 schools). The distributions are estimated according to Parzen method [8] and are denoted by $\left\{H_{f}\left(I_{s}\right)\right\}_{f=1: 20, s \in \Omega}$ where $f$ is the feature index.

According to these acoustic features, we compute the similarity between two images as follows:

$$
\operatorname{Dist}\left(I_{k}, I_{s}\right)=\sum_{f=1}^{20} K L\left(H_{f}\left(I_{k}\right), H_{f}\left(I_{s}\right)\right)
$$

where $K L$ is the Kullback-Leibler divergence [9].

We use this distance within a fast marching algorithm [6] to compute the weights $\left\{w_{k}\right\}_{k=1: K}$. Fast marching algorithm is a numerical technique designed to track the evolution of interfaces. It aims at marching outward, computing distances from mesh points to the interface. The key step is to compute the distance to the next nearest mesh point. Then the front moves further outward with velocity $F$ according to the Hamilton-Jacobi equation:

$$
|\nabla T|=1 / F
$$

where $F>0$ is the front moving speed and $T$ is the travel time. $1 / F$ and $T$ may also be interpreted as travel cost and intrinsic instance respectively. The time $T$ at a grid point $I_{s}$ depends on the punctual velocity $F\left(I_{s}\right)$ and also on the spatial distance between the point $I_{s}$ position and the starting point position.

We propose to use $K$ fast marching processes, each initialized by an identified image $I_{k}$ and with a velocity $F_{k}\left(I_{s}\right)=\operatorname{Dist}\left(I_{k}, I_{s}\right)$.

Each propagation scheme $k$ estimates the function $T_{k}\left(I_{s}\right)$ for each grid point $I_{s}$. Then $\left\{T_{k}\left(I_{s}\right)\right\}$ are normalized and used as the weights for the fish abundance estimation. 


$$
w_{k}\left(I_{T}\right)=\frac{G\left(T_{k}\left(I_{T}\right)\right)}{\sum_{i=1}^{K} G\left(T_{i}\left(I_{T}\right)\right)}
$$

where $G$ is a radial kernel.

\section{Results and discussion}

We applied the proposed method on both synthetic and real data.

\section{A. Synthetic data}

Synthetic data are generated from real fish school features as follows:

- We estimate fish features (e.g., fish density, target strength, depth, school shape as a set of ellipsoidal volumes);

- We simulate several fish school using OASIS acoustic fish school simulator [10], which generates acoustic echograms from a given echosounder and vessel setting and the given school characteristics;

- We fix a target fish proportion grid;

- We associate to each grid point an echogram comprising schools randomly selected from the simulated dataset according to set class proportions. The random selection induces a 'noisy' proportion map.

During the test, we suppose known the proportions in some images selected randomly (identified images) and then, we apply the proposed interpolation method to estimate the abundance on the rest of images.

We show two grid examples that are simulated with real school dataset from Ifremer [7]:

- a four-region case for which each region is composed of a single specie: 'Sardine', 'Anchovy', 'Horse Mackerel' and 'Chinchard' ;

- a six-region case for which each region is a mixture of mainly two species.

In figures 3 and 4 , we show the initial data grids respectively a $128 \times 128$ and $250 \times 250$ squared images (each pixel refers to an acoustic image). For each identified image we show the fish abundance $\Pi_{k}$.

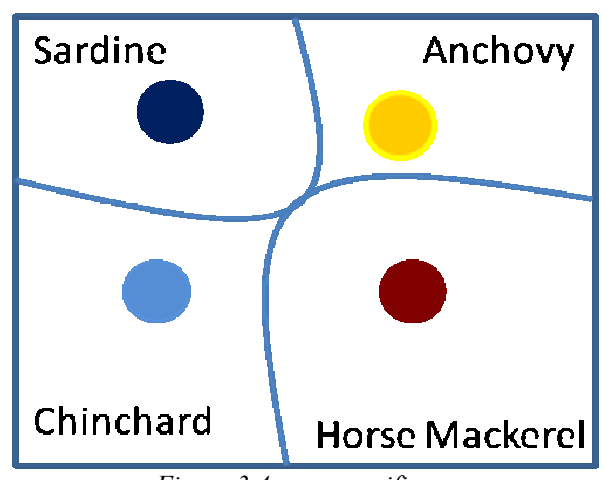

Figure 3.4 monospecific case

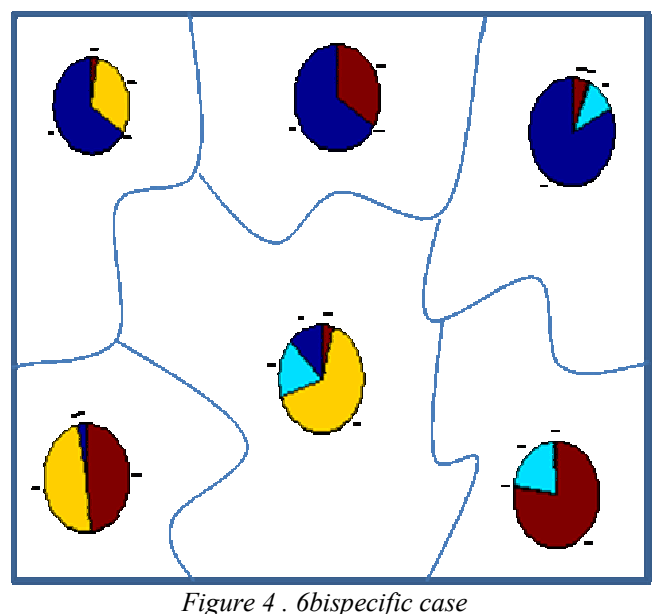

In figure 5, we show the simulated proportion map for the sardine specie for the six-region case. It might be noticed that the simulation procedure results in "noisy" proportion maps.

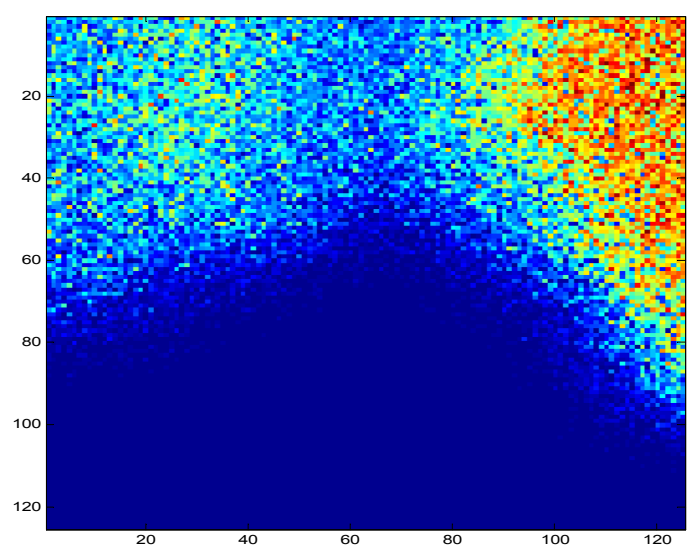

Figure 5. Sardine proportion map

In table I, we give the estimation error mean for each specie and for both cases.

The error is computed as follows:

$$
\tau_{j}=\sum_{T \in \Omega}\left(\Pi_{j, s}\left(I_{s}\right)-\Pi_{j, e}\left(I_{s}\right)\right)
$$

where $\Pi_{j, s}\left(I_{s}\right)$ respectively $\Pi_{j, e}\left(I_{s}\right)$ are the simulated and the estimated proportion for specie $j$ on the image $I_{s}$.

In figure 6 we show the sardine proportion estimation error map for the six-region case.

\begin{tabular}{|l|c|c|c|c|}
\hline & Sardine & Anchovy & Chinchard/Sprat & Mackerel \\
\hline 4-class & $10 \%$ & $20 \%$ & $10 \%$ & $15 \%$ \\
\hline 6-class & $25 \%$ & $20 \%$ & $25 \%$ & $20 \%$ \\
\hline
\end{tabular}

Table I. Estimation error rate for each specie proportion and for each example. 


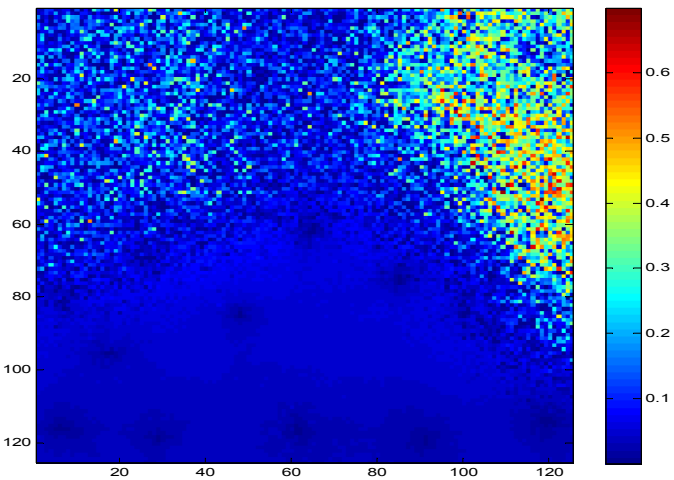

Figure 6.Sardine estimation error map

\section{B. Real data}

Figures 7-10 show the interpolation results of the fish proportions ('Anchovy', 'Sardine', 'Horse Mackerel' and 'Sprat') for the French fisheries-acoustic survey of IFREMER performed on spring 2000 over the French shelf of Biscay performed with the RV "Thalassa". Trawl catch positions are presented in red circles.

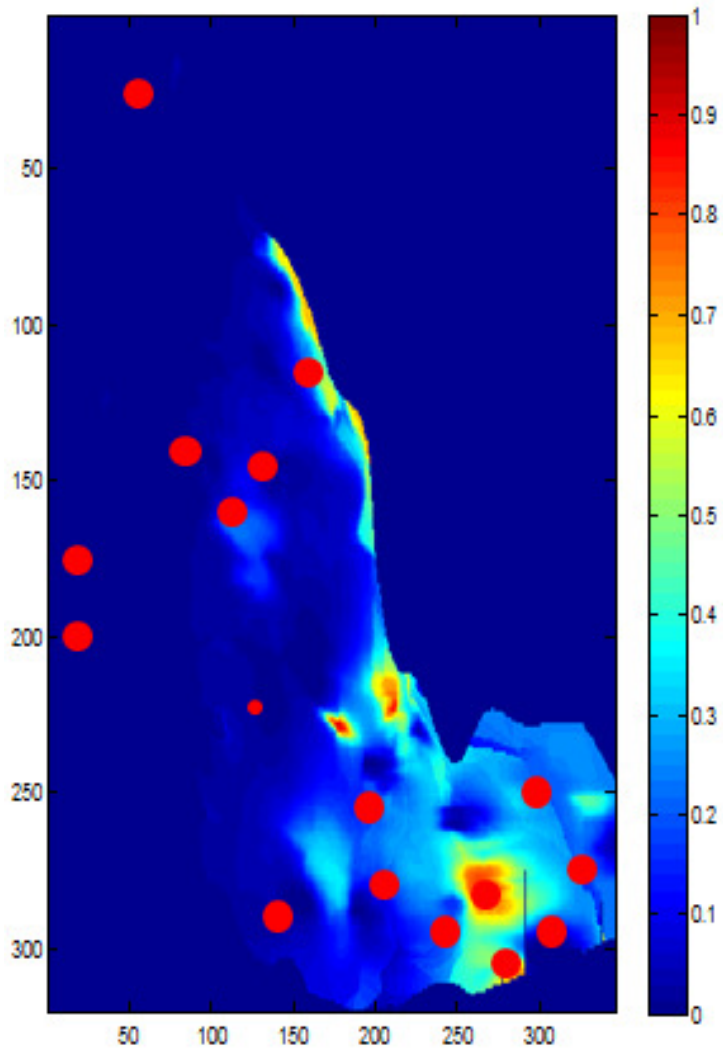

Figure 7. Anchovy proportion map

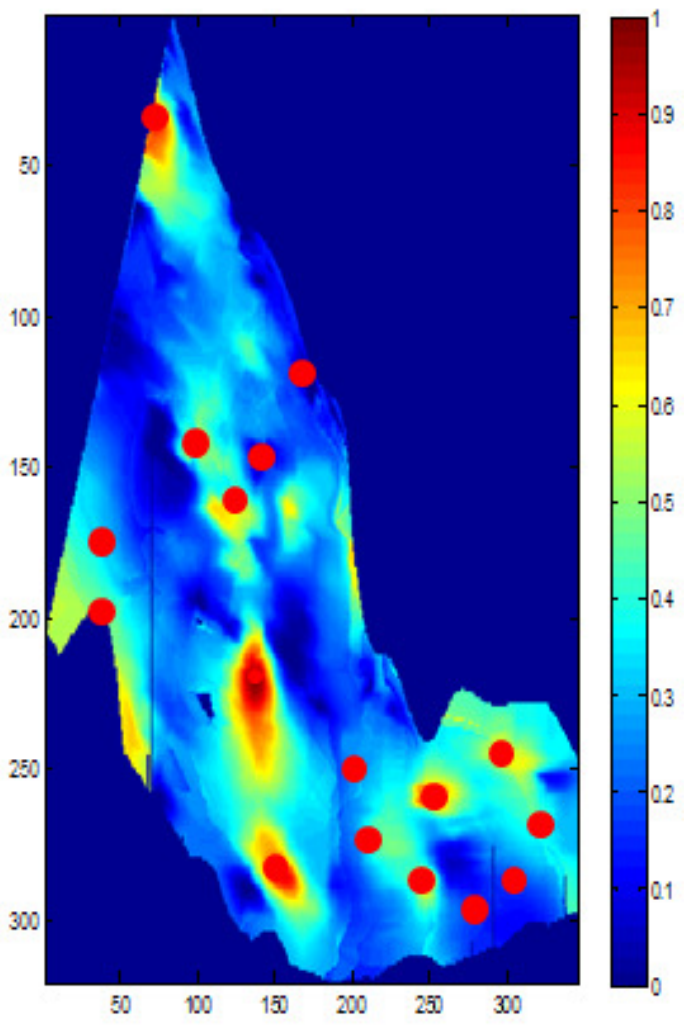

Figure 8. Sardine proportion map

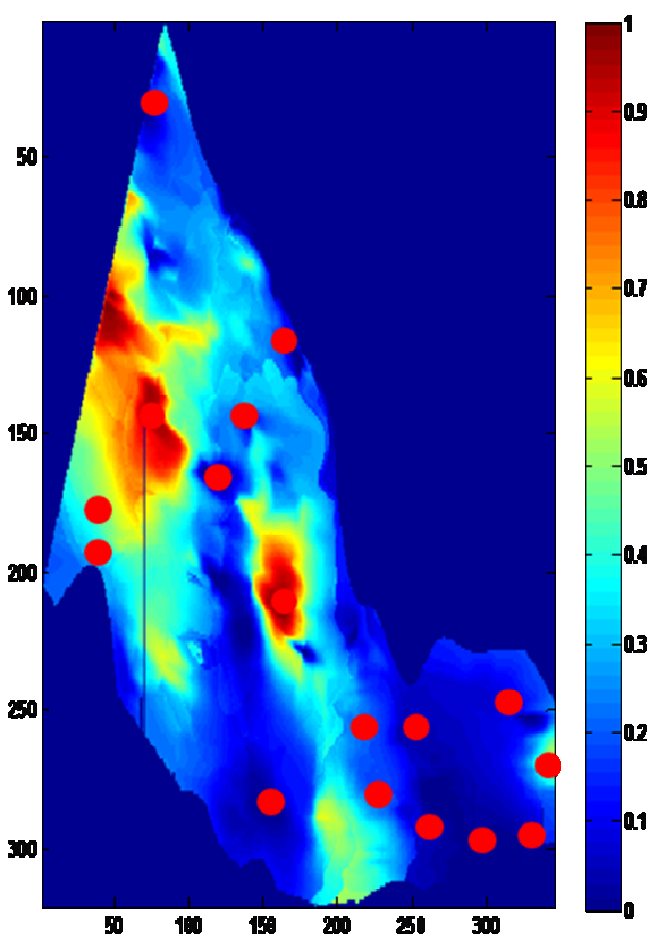

Figure 9. Horse Mackerel proportion map 


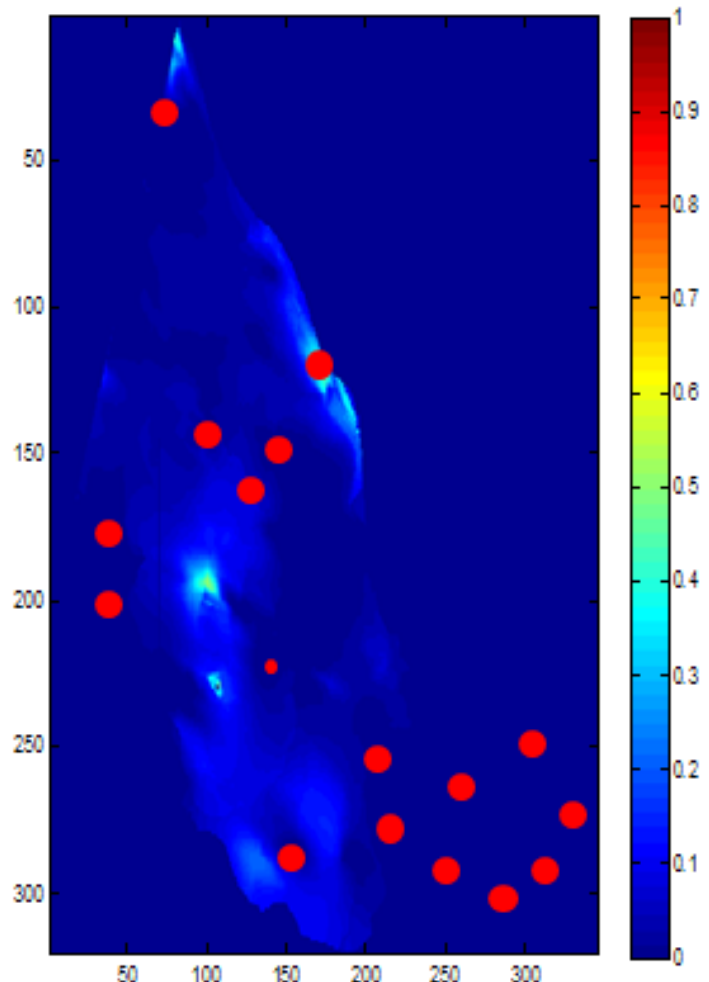

Figure 10. Sprat proportion map

\section{Conclusion}

We proposed a new for fish proportion estimation from acoustic features and some trawl catches. The interpolation is based on a weighting scheme computed from fast marching algorithm. The method allows taking into account both of the acoustic similarity between images and also the spatial distance between non identified echograms and the trawled areas.

In future work other global features extracted directly from the echogram and not only on the schools extracted from it, can be investigated such as the echogram backscatter distribution and texture.

\section{References}

[1] Hammond, T.R., and Swartzman, G.L. A general procedure for estimating the composition of fish school clusters using standard acoustic survey data. Ices Journal of Marine Science 58(6): 1115-1132, 2001.

[2] Haralambous, J., and Georgakarakos, S. Artificial neural netorks as a tool for species identification of fish schools. ICES Journal of Marine Science. 53(2): 173-180, 1996.

[3] Petitgas, P., Masse, J., Beillois, P., Lebarbier, E., and Le Cann, A. Sampling variance of species identification in fisheries-acoustic surveys based on automated procedures associating acoustic images and traw hauls. ICES Journal of Marine Science 60(3): 437-445, 2003.

[4] Hammond, T.R., Swartzman, G.L., and Richardson, T.S. Bayesian estimation of fish school cluster composition applied to a Bering Sea acoustic survey. ICES Journal of Marine Science 58(6): 1133-1149, 2001.
[5] A.Weil, C. Scalabrin and D. Diner, MOVIES-B: an acoustic detection description software: application to shoal species classification, Aquatic Living Resources, 6:255-267, 1993.

[6] J.A. Sethian, Fast Marching Methods and Level Set Methods for Propagating Interfaces, Von Karman Institute Lecture Series, Computational Fluid Mechanics, 1998.

[7] Scalabrin, C., Diner, N., Weill, A., Hillion, A., and Mouchot, M. Narrow band acoustic identification of mono specific fish shoals. ICES Journal of Marine Science, 53: 191-188, 1996.

[8] Parzen. P. On the Estimation of a Probability Density Function and the Mode. Annals of Mathematical Statistics. Vol.33, 1065-1076, 1962.

[9] S.Kullback, On information and sufficiency, The Annals of Mathematical Statistics, Vol.22, Wiley, New York, 1951.

[10] Diner, N. Correction on school geometry and density: approach based on acoustic image simulation. Aquatic Living Resources 14(4): 211-222, 2001 